despite its name, gives off infrared light when it fluoresces). Although ICG has been clinically approved for years, it stops fluorescing when it conjugates (or covalently binds) to protein, making chemists reluctant to use it. Kobayashi reasoned that this silencing might be an advantage: he attached ICG to an antibody that both silenced the fluorescence and carried the fluorophore to the target of interest. Once the antibody was bound to the target and brought into the cell, ICG detached, becoming fluorescent ${ }^{2}$. The fact that this advantage was overlooked, says Kobayashi, is an example of how chemists' thinking can hold back development of activatable fluorophores; chemists work with such labels in solution and aren't trained to think about how fluorophores will act inside an animal's body, he says. "We pick up a lot of trash."

Probes are the workhorses of the optical imaging system, and researchers such as
Kobayashi and Piwnica-Worms are coming up with increasingly creative ways to get them into the right places and to detect them once they are there. Such advances in technology promise that researchers will soon be able to image more processes in more types of cells (see 'Probe progress'). Partly as a result, expectations for optical approaches have been rising steadily, says William McLaughlin of Carestream Molecular Imaging in Woodbridge, Connecticut, which

\title{
PROBE PROGRESS
}

A big limitation of molecular imaging in whole animals is how hard it is to measure multiple signals, says Sanjiv Sam Gambhir, who directs the Molecular Imaging Program at Stanford in California.

"Unlike our colleagues who remove tissues or blood samples from animals and can analyse many, many things, we're very limited in how many signals we can get simultaneously," he says. "In this field, it's not so much about the instruments but about the abilities of the imaging agents."

These agents are not always quick to develop, says Michael Olive, vice-president of science and technology at LI-COR Biosciences in Lincoln, Nebraska, which sells optical instruments and agents. It's not simply a matter of attaching a fluorophore to a ligand, he says. Before trying a new probe in an animal, for example, LI-COR uses cell lines to see whether increasing the concentration of the unlabelled ligand displaces the labelled one; if not, the label is not binding specifically and can't be used for further studies. If so, the company does more tests, then an extensive autopsy to look for any traces of the label. Without this precaution, says Olive, unanticipated labelling could wreck an experiment. "It probably takes as much as 4-6 months to have confidence that the marker is really doing what you think it's doing."

A problem for the optical techniques used to detect these probes is that most signals cannot travel very far through tissue. Some optical techniques are being used clinically, but they are restricted to organs such as the breast, bladder or stomach, where a detection device can be placed in or close to the site. By contrast, labels developed for PET or MRI for whole-body studies in patients can

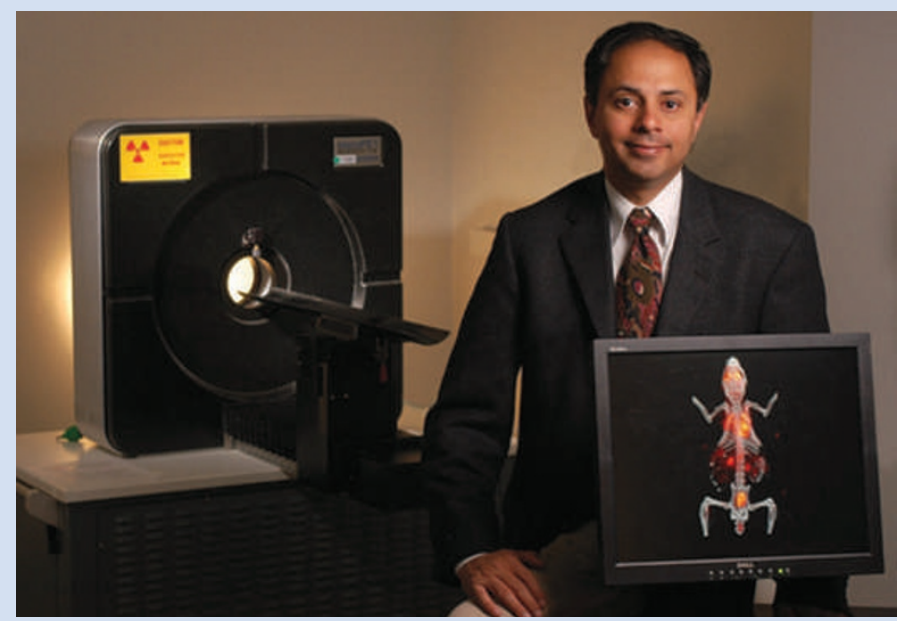

Sanjiv Sam Gambhir is working to link together various imaging modalities.

be readily used in animals. "Most of the optical technologies are never going to be translated to humans," says Michael Welch at Washington University in Saint Louis, who uses PET to study glucose metabolism in mouse models of diabetes.

Gambhir is doing his part to develop new labels. He began his career in imaging at the University of California, Los Angeles, as a teenage assistant to Michael Phelps, the co-inventor of PET. This year, he mutated luciferase to emit signals at a longer wavelength, allowing it to penetrate farther through tissues. He has also created imaging agents that produce light when acted on by particular cues, photoacoustic agents that convert light signals to sound, as well as nanoparticles that enhance Raman peaks - characteristic shifts in wavelength frequency - and so may allow detection of as many as ten spectral signals within an animal. Current versions of the particles are too large to enter cells, but Gambhir says that they can be made smaller. Nevertheless, he says, large size can be an advantage. "Delivery is harder because they are bigger, but you also get more signal because they are bigger." Several groups are working on imaging agents that are activated by one kind of energy but emit another. Fluorescent probes, for example, must be activated by light to emit light. That creates a background signal that limits the resolution of the resulting image. Optimized dyes and software can minimize the problem, but start-up nanobiotechnology firm Zymera in San Jose, California, is working on quantum dots that can bypass it. These dots are activated by high-energy, short-wavelength bioluminescent light produced within the body by luciferase coating the nanoparticle, but, rather than emitting a similar wavelength, they emit the near-infrared light best able to penetrate tissues. The dots could be used in machines optimized for fluorescence.

But getting a high-resolution image from light coming from deep within an animal, even a small one, is still a problem, says Vasilis Ntziachristos, who directs the molecular imaging department at the Technical University of Munich in Germany. Not only are many photons absorbed before they reach the charge-coupled devices that detect them, the way that tissues scatter light makes lost data extremely difficult to reconstruct. Ntziachristos is developing photoacoustic agents that create sound signals when they absorb light, and so allow signals to travel farther through tissues with less scattering. He has shown that organic dyes and fluorescent proteins can be detected with ultrasound and used for wholebody three-dimensional imaging of animals such as fruitflies, zebrafish and mice. The resolution is around 10-100 micrometres, potentially orders of magnitude better than that achievable by light, he says.

To create the signal, lasers transmit nanosecond pulses of light onto a chromophore, causing it to heat up and cool down. The resulting contraction and expansion generates a sound wave that can not only be detected but also traced to a particular depth. "When taking a photograph of an animal, what you see is the light that has been reflected or scattered through the surface," says Ntziachristos. That makes for images with poor resolution, he explains. "Photoacoustic tomography gets the resolution back."

But detecting sound rather than light is not a simple switch. Figuring out where to place the sound detectors is more complicated than setting up a home stereo system. Then there's the problem of a coupling medium, says Ntziachristos. His acoustics systems don't work as well in air, for instance. "But more importantly," he says, "you need mathematics." The data have to be processed, and variations induced by animal tissue and hardware must be accounted for. "Without the reconstruction software, you cannot produce volumetric images," he says. 